\title{
Lifetime and aging of chromatography resins during biopharmaceutical manufacture
}

Mauryn C. Nweke ${ }^{\text {a }}$, Anurag S. Rathore ${ }^{\text {b }}$, Daniel. G. Bracewell* a

a Department of Biochemical Engineering, University College London, Bernard Katz Building, Gower Street, London WC1E 6BT, United Kingdom

${ }^{\mathrm{b}}$ Department of Chemical Engineering, IIT Delhi, Hauz Khas, New Delhi, Delhi 110016, India

\section{Abstract}

Poor understanding of the events leading to column aging, make it difficult to monitor column lifetime. The lack of established procedures in this area has made it difficult to establish industry standards. Therefore, it is important to understand resin aging mechanisms and potential techniques to monitor column aging during operation.

Key words: Chromatography resin aging; Chromatography resin lifetime; Chromatography resin aging mechanisms; Fouling characterisation techniques

*Correspondence: d.bracewell@ucl.ac.uk (D. G. Bracewell) 


\section{The importance of understanding resin aging}

Resin aging is a gradual deterioration of the performance attributes pertaining to chromatography media. It occurs due to repeated exposure to a range of bioprocessing conditions. Changes in dynamic binding capacity (DBC) and yield are predominantly used to monitor chromatographic performance over time, however, due to the need to reuse these resins for a large number of cycles, it is important to understand the performance characteristics less commonly explored, such as mechanical and structural aging, and how these link to those attributes more closely monitored.

A poor understanding of column aging directly impacts on two key areas - cost and regulation. Chromatography is the most expensive part of downstream processing. In a typical mAb process, as much as $60 \%$ of downstream costs come from chromatography alone and the cost of a typical Protein A resin (which is commonly used as the first of multiple chromatographic steps) is $50 \%$ higher than the cost of other types of chromatographic media, such as ion exchangers [1]. Aside from economic implications, the increased product titres achieved within the last few years have led to a heavier burden on downstream processing. In addition, the Quality by Design (QbD) and Process Analytical Technology initiatives call for increased process and product understanding to ensure that each process consistently meets precisely defined quality attributes. This has led to increased research in factors that define the critical quality attributes (CQAs) of resin aging and linking them to their corresponding critical process parameters (CPPs), resulting in a thorough and systematic approach to characterise the process parameter space. 


\section{Mechanisms of aging}

Literature suggests that process conditions determine the rate of performance decay. An unsuitable packing process, for example, subjects the column to excess compression during bioprocessing, and thereby reduces the expected lifetime of the resin [2]. However, most reports on resin aging tend to largely focus on quantifying the loss in performance characteristics (Table 1). Whilst reduced DBC and yield are often measured concurrently, reductions in DBC are exclusively related to ligand deterioration factors, whilst reductions in yield may also be related to additional factors [3]. A decline in ligand performance may occur for a number of reasons such as, ligand leaching off of the matrix due to chemical or mechanical stresses, or ligand occlusion by non-target molecules, which reduces the availability of ligand active sites for target molecules and therefore binding activity. Inactivation of the ligand may also occur due to possible changes in ligand chemistry due to process conditions [4]. This has led to the engineering of novel ligands that promote higher binding capacities [5]. These mechanisms indicate that the resin aging can be assigned to two areas: capacity loss - (ligand leaching, ligand inactivation and ligand occlusion) and packed bed failure (matrix fouling, matrix degradation/deformation.)

A recent study involving fouling of Protein A resin reported that the dominant mechanism of fouling is the non-specific adsorption of the feed material components on resin, signalling that over a large number of cycles, pore diffusion becomes the rate limiting step [6]. 


\section{Monitoring the aging of chromatography resins}

Several researchers have investigated the contribution of fouling to the decline in column performance and proposed techniques to observe this phenomenon [4], [5]. Commonly reported techniques include the use of different microscopy and spectroscopy procedures [6], [7] (Figure 1). However, only a few studies have employed these techniques in-situ or in real time. Boulet-Audet and colleagues [9] coupled affinity chromatography and Fourier transform infrared (FTIR) spectroscopy by embedding an attenuated total reflection (ATR) sensor inside a micro- scale column while measuring the UV $280 \mathrm{~nm}$ and conductivity. Their approach quantified the in-column protein concentration in the resin bed, determined protein conformation, and also detected Protein A ligand leaching during CIP. Pathak and colleagues [10] developed a fluorescence-based real time monitoring tool of fouling during chromatographic operation. This tool could detect changes in protein A ligand densities, foulant concentration build-up, and foulant deposition during cation exchange chromatography.

\section{Treatments and troubleshooting}

A number of methods have been proposed to improve resin lifetime and performance. Revisions to clean-in-place (CIP) and regeneration conditions can extend resin lifetime. Researchers have investigated a number of CIP procedures optimised for protein A chromatography using design of experiments (DoE) approach. After exposure to typically higher concentrations of CIP reagents for an extended time period MabSelect Sure (a type of protein A resin) retained $90 \%$ of its original capacity and the addition of sodium chloride provided a stabilizing effect on the resin. More recently, researchers developed a highthroughput method for screening for CIP conditions on MabSelect Sure and reported similar observations[11]. 
Improvements to clarification strategies as well as alterations to process feed material and process conditions, such as flow rate and mobile phase viscosity, can also act as troubleshooting techniques to extend resin lifetime. Poorly clarified suspensions require proportionally larger filters, however increased clarification leads to greater column capacity. This is an increasing issue associated with the intensification of upstream operations driving interest in clarification approaches to ameliorate damage to protein A chromatography based on precipitation, flocculation and depth filter design.

Choosing a suitable adsorbent for the target protein is important. The degree of agarose-based resin cross-linking has been reported to contribute towards both the chemical and mechanical stability of the resin. It is widely recognised that too high a degree of cross-linking causes mass transfer limitations, resulting in reduced resin functionality. Further enhancements include improvements to ligand stability. These include protein-structure-based designs, which involve retrieving structural information about the target protein and subsequently identifying a potential binding site [5]. Protein-function-based designs, involving identification of specific functional groups on the protein and identifying a binding site, offer another possibility. 


\section{Concluding remarks}

There is a need to understand the mechanisms of aging that lead to the decline in mechanical and structural performance characteristics. Literature suggests that resin aging can be assigned to two main areas of capacity loss and packed bed failure. Currently there are a few novel techniques employed at research scale used to monitor the structural aging of resins via matrix fouling and ligand leaching characterisation. However, there is a need to develop techniques that specifically monitor the mechanical deterioration of these resins over time or further still, establish a link between the observed structural decay and mechanical performance. There are a limited number of techniques at research scale that address this directly, such as work carried out by Nweke and colleagues [12] however, these techniques have yet to be employed for aging studies and the link to other performance parameters such as DBC is yet to be made.

Extensive research continues in this area and the improved understanding of column aging mechanisms, successful implementation of these techniques at lab-scale as well as regulatory pressures for better process characterisation may all serve as a driving force towards the implementation of such techniques during industrial-scale separation. 
Table 1 Parameters associated with the quantification of resin lifetime [13]

Measurable parameters for determining chromatography resin lifetime

Purity of product

Impurities (e.g. DNA)

Pressure

Product yield

Elution profile

Regeneration profile

Loading profile

Voidage (inter/intra particle porosity)
Bed height

Dynamic/static binding capacity

Packing integrity (HETP)

Leachables

Ionic capacity (IEX)

Appearance (microscopy)

Productivity

Adsorption isotherms 
Protein A ligand

40-45 kDa
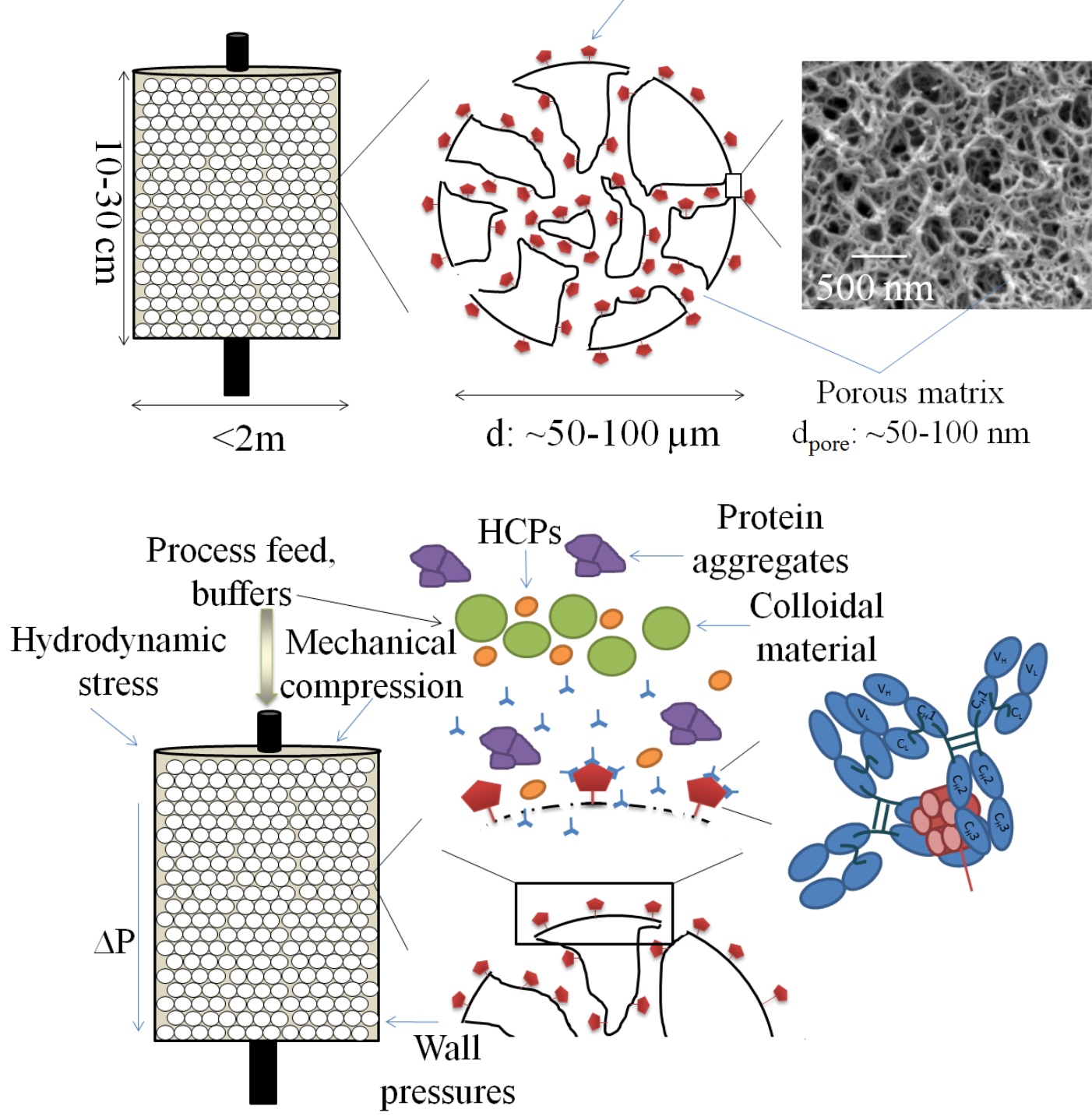

Ligand leaching

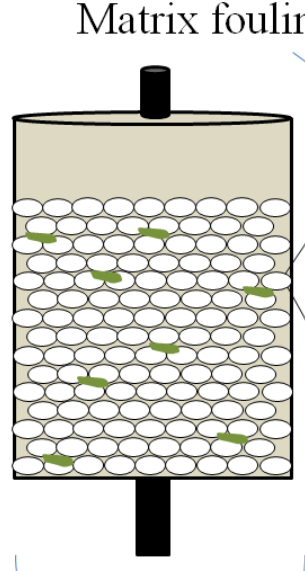

Changes to packed bed structure

Due to column plugging and compression

\section{Ligand occlusion}

Ligand inactivation
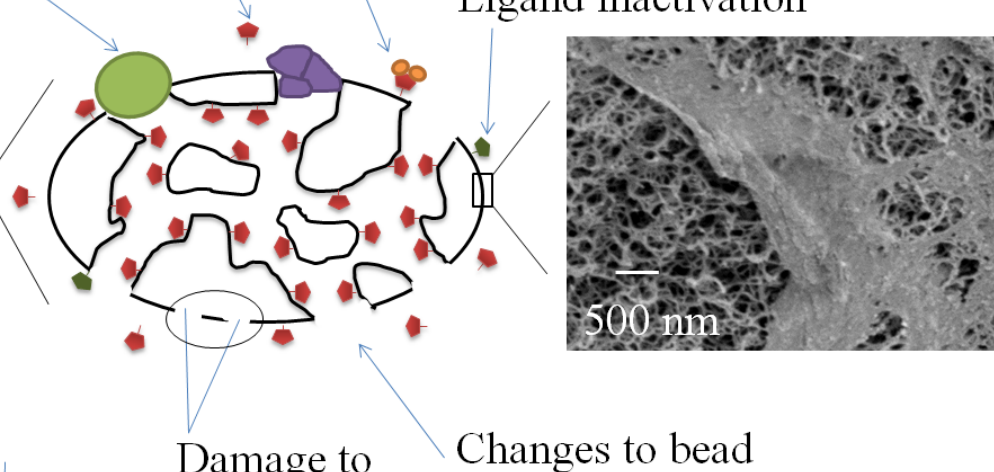

Changes to bead morphology

\section{matrix}

Potentially

leading to resin

fines 
Figure 1 - (A) showing size of typical large-scale column, schematic of resin structure and scanning electron microscopy (SEM) image of protein A resin prior to processing. (B) showing conditions that the column and beads are exposed to. (C) showing the impact of process conditions over repeated cycles of exposure to the column and bead and an SEM image showing fouling of the bead surface. 
Bibliography

[1] A. Rathore, M. Pathak, G. Ma, and D. Bracewell, "Re-use of Protein A Resin: Fouling and Economics," BioPharm Int., vol. 28, no. 3, 2015.

[2] M. Dorn, F. Eschbach, D. Hekmat, and D. Weuster-Botz, "Influence of different packing methods on the hydrodynamic stability of chromatography columns," $J$. Chromatogr. A, 2017.

[3] A. T. Hanke and M. Ottens, "Purifying biopharmaceuticals: knowledge-based chromatographic process development," Trends Biotechnol., vol. 32, no. 4, pp. 210$220,2014$.

[4] S. Zhang et al., "Structural and functional characteristics of virgin and fouled Protein A MabSelect resin cycled in a monoclonal antibody purification process.," Biotechnol. Bioeng., pp. 1-29, Jul. 2015.

[5] T. Pabst et al., "Engineering of novel Staphylococcal Protein A ligands to enable milder elution $\mathrm{pH}$ and high dynamic binding capacity," J. Chromatogr. A, vol. 1362, no. 3, pp. 180-185, 2014.

[6] M. Pathak and A. S. Rathore, "Mechanistic understanding of fouling of protein A chromatography resin,” J. Chromatogr. A, vol. 1459, pp. 78-88, 2016.

[7] E. J. Close, J. R. Salm, T. Iskra, E. Sørensen, and D. G. Bracewell, "Fouling of an anion exchange chromatography operation in a monoclonal antibody process: Visualization and kinetic studies.," Biotechnol. Bioeng., vol. 110, no. 9, pp. 2425-35, Sep. 2013.

[8] K. Lintern, M. Pathak, C. Smales, K. Howland, A. Rathore, and D. Bracewell, "Residual on column host cell protein analysis during lifetime studies of protein A chromatography," J. Chromatogr. A, vol. 1461, pp. 70-77, 2016.

[9] M. Boulet-Audet, S. G. Kazarian, and B. Byrne, "In-column ATR-FTIR spectroscopy to monitor affinity chromatography purification of monoclonal antibodies," Sci. Rep., vol. 6, no. 1, p. 30526, 2016.

[10] M. Pathak, K. Lintern, V. Chopda, D. G. Bracewell, and A. S. Rathore, "Fluorescence 
based real time monitoring of fouling in process chromatography.," Sci. Rep., vol. 7, no. October 2016, p. 45640, 2017.

[11] A. Grönberg, M. Eriksson, M. Ersoy, and H. J. Johansson, "A tool for increasing the lifetime of chromatography resins," MAbs, vol. 3, no. 2, pp. 192-202, Oct. 2011.

[12] M. C. Nweke, R. G. McCartney, and D. G. Bracewell, "Mechanical characterisation of agarose-based chromatography resins for biopharmaceutical manufacture," $J$.

Chromatogr. A, vol. 1530, pp. 129-137, Dec. 2017.

[13] A. Rathore and G. Sofer, Life Span Studies for Chromatography and Filtration MediaProcess Validation in Manufacturing of Biopharmaceuticals, 1st ed. Taylor \& Francis: Boca Raton, FL, 2005. 